\title{
THE ROLE OF DAILY CO-CARBIDOPA THERAPY IN CHRONIC RESTLESS LEG SYNDROME AND ITS ASSOCIATION WITH AUGMENTATION IN A STUDY IN TERTIARY CARE CENTRE IN SOUTH INDIA
}

\author{
Mohanakkannan $S^{1}$, Sowmini P. R², Sakthi Velayutham³, Malcolm Jeyaraj', Mugundhan Krishnan', Arunan $S^{6}$ \\ ${ }_{1}^{1}$ Postgraduate Student, Department of Neurology, Stanley Medical College and Hospital, Chennai, Tamilnadu, India. \\ ${ }^{2}$ Assistant Professor, Department of Neurology, Stanley Medical College and Hospital, Chennai, Tamilnadu, India. \\ ${ }^{3}$ Assistant Professor, Department of Neurology, Stanley Medical College and Hospital, Chennai, Tamilnadu, India. \\ ${ }^{4}$ Assistant Professor, Department of Neurology, Stanley Medical College and Hospital, Chennai, Tamilnadu, India. \\ ${ }_{5}^{5}$ Associate Professor, Department of Neurology, Stanley Medical College and Hospital, Chennai, Tamilnadu, India. \\ ${ }^{6} \mathrm{HOD}$, Department of Neurology, Stanley Medical College and Hospital, Chennai, Tamilnadu, India.
}

ABSTRACT
BACKGROUND
Co-carbidopa was the first drug introduced for restless leg syndrome. But its use is not advocated much due to increased incidence
of augmentation amongst the users. The first dopaminergic drug that was used in the treatment of RLS was co-carbidopa. However,
it was noted in subsequent studies that there was a decline in the efficacy of co-carbidopa and there was an increase in the
frequency, duration and intensity of symptoms with increased duration of co-carbidopa therapy which was termed as
"augmentation". We wanted to define the role of co-carbidopa therapy in Chronic Restless Leg Syndrome and its association with
augmentation.

\section{METHODS}

30 patients of chronic RLS were selected according to the IRLSSG criteria. They were treated with co-carbidopa. Their IRLSSG scores were monitored at baseline, 2 months and 6 months. Every month, the patients were monitored according to Max Planck Institute criteria for development of augmentation. If the patients develop augmentation, they were treated with Gabapentin. The IRLSSG scores were compared for treatment efficacy.

\section{RESULTS}

10 of $30(33.3 \%)$ patients developed augmentation. The remaining 20 patients had statistically significant reduction in IRLSSG score at 2 months $(\mathrm{p}=0.003)$ and 6 months $(\mathrm{p}=0.009)$.

\section{CONCLUSIONS}

Co-carbidopa may be an effective therapy in chronic daily restless legs syndrome as suggested by the good response in our study. The incidence of augmentation amongst South Indian population may not be as high as the western literature. Restless legs syndrome can be easily mistaken for small fibre neuropathy of Diabetes Mellitus and subsequent treatment with tricyclic antidepressants may worsen their symptoms.

\section{KEY WORDS}

Restless Leg Syndrome, Augmentation, Carbidopa in RLS

HOW TO CITE THIS ARTICLE: Mohanakkannan S, Sowmini PR, Velayutham S, et al. The role of daily co-carbidopa therapy in chronic restless leg syndrome and its association with augmentation in a study in tertiary care centre in South India. J. Evolution Med. Dent. Sci. 2019;8(23):1816-1819, DOI: 10.14260/jemds/2019/399

\section{BACKGROUND}

Restless legs syndrome is a neurological sensorimotor disorder that is characterized by an irresistible urge to move the lower limbs at rest, and it is frequently accompanied by nocturnal parasthesiae. ${ }^{1}$ This has been a common disorder affecting sleep and health for decades. ${ }^{2-4}$ Chronic restless legs syndrome is defined as restless legs syndrome with symptoms that would occur on average at least twice weekly for the past year when not treated.

'Financial or Other Competing Interest': None.

Submission 26-03-2019, Peer Review 25-05-2019,

Acceptance 31-05-2019, Published 10-06-2019.

Corresponding Author:

Dr. Sowmini P. R,

Assistant Professor

Department of Neurology,

Stanley Medical College and Hospital,

Chennai, Tamilnadu, India.

E-mail: drprsowmini@yahoo.co.in

DOI: $10.14260 /$ jemds/2019/399

\section{(c) (i) $(3)$}

Prevalence of restless legs syndrome is $1.9-4.6 \%$ of European and North American general populations in epidemiological studies. 5 The first dopaminergic drug that was used in the treatment of RLS was co-carbidopa. However, it was noted in subsequent studies that there was a decline in the efficacy of co-carbidopa and there was an increase in the frequency, duration and intensity of symptoms with increased duration of co-carbidopa therapy which was termed as "Augmentation ". The first line of treatment nowadays is dopamine agonists. Augmentation is noted to develop in both co carbidopa and dopamine agonist, but more often with co-carbidopa. Allen and Early identified and defined " Augmentation " by the following four possible features:(a) Anticipation at time of onset of symptoms to earlier in afternoon (b) Expansion of symptoms to upper limbs, (c) Shorter latency to symptoms while at rest and (d) Overall increase in the symptom intensity ${ }^{6}$ Augmentation can be a significant obstacle for physicians aiming to ameliorate symptoms of restless legs syndrome and it is treated by decreasing the dose of co-carbidopa/dopaminergic agonists or by addition of $\alpha 2 \delta$ ligands (Gabapentin)/benzodiazepines. 
Since the advent of dopaminergic agonists (Pramipexole and Ropinirole) and due to the increased incidence of augmentation, co-carbidopa is advocated only as second line therapy in restless legs syndrome with intermittent symptoms. There are only a few studies on the incidence of augmentation secondary to co-carbidopa therapy in Indian population. The study aims to define the role of co-carbidopa therapy in chronic daily restless legs syndrome and the incidence of Augmentation in study population in South India.

\section{METHODS}

This study was performed as a single centred, uncontrolled clinical trial over a period of 1 year. All patients who satisfied the criteria of Restless leg syndrome who attended Neurology OPD in Stanley medical College and hospital were included in the study. Before including any patient in the study, a voluntary written informed consent was taken from the patient. 30 patients above the age of 18 years who had evidence of restless legs syndrome according to IRLSSG (International Restless leg syndrome society guidelines) criteria $^{7}$ were selected. Only the patients with chronic restless legs syndrome (Symptoms when not treated would occur on average at least twice weekly for the past year) were included in the study. Patients who fell in the group of intermittent restless legs syndrome according to IRLSSG criteria (Symptoms when not treated would occur on average $<2$ /week for the past year, with at least five lifetime events) were excluded from the study even if they met the inclusion criteria for restless legs syndrome. For all the patients included in the study baseline complete blood count, renal function tests, serum ferritin, fasting blood sugar, post prandial blood sugar and $\mathrm{HbA1c}$ were performed. A nerve conduction study was done for all the patients in the study group to evaluate for presence of underlying neuropathy. Patients with both chronic Primary and chronic Secondary restless legs syndrome (Secondary to Diabetic neuropathy, renal failure, Iron deficiency anaemia, Parkinson disease) were included in the study. Co-carbidopa was started for all the selected patients. The starting dose of Co-carbidopa was $(100 \mathrm{mg}+10 \mathrm{mg})$ in two divided doses and was titrated up to a maximum dose of ( $300 \mathrm{mg}+30 \mathrm{mg}) /$ day in three divided doses. The criteria for up titration of syndopa was to eliminate all symptoms of restless legs syndrome in the patient and once symptoms are alleviated to keep the same dose as maintenance. Patients were reviewed every month for over a period of 6 months. Dose titration was done throughout the study period to help alleviate the restless legs syndrome symptoms in patients. IRLSSG scores were monitored at baseline, 2 months and 6 months. The patients were enquired about the presence of symptoms of Augmentation every month using MPI (Max Planck Institute) criteria. ${ }^{8}$ If patients developed Augmentation, co-carbidopa was stopped and replaced with Gabapentin. Gabapentin was started at $300 \mathrm{mg}$ bedtime dose and titrated according to patient's restless legs syndrome symptoms.

\section{Statistical Methods}

For numerical variables like age they are expressed in means and standard deviations and rest of the variables are expressed in proportions. For continuous variables paired $t$ test is used to find the association. $\mathrm{P}$ value $<0.05$ was considered as statistically significant. SPSS software version 17 was used for the calculations.

\section{Sample Selection}

30 patients were selected according to the IRLSSG criteria. Of the 30 patients selected, 20 were females and 10 were males. Mean age of symptom onset was 52 years. 24 of the 30 patients had Type 2 Diabetes Mellitus, all of whom had underlying neuropathy as evidenced by clinical examination and Nerve conduction study according to the AAN, AANEM and AAPMR guidelines for diagnosis of Diabetic Neuropathy ${ }^{9}$ Mean HbA1c was 8.5 in patients with Diabetes. Renal function tests were normal in all the patients. The remaining three patients who did not have Type 2 Diabetes Mellitus did not have any other co- morbidities and were included as chronic idiopathic restless legs syndrome.

\section{RESULTS}

10 out of 30 patients $(33.3 \%)$ of patients treated with cocarbidopa developed Augmentation according to Max Planck Institute criteria. Of the ten patients who developed augmentation, 6 had underlying Diabetic neuropathy and 4 patients had primary restless legs syndrome. All the five patients who developed Augmentation were on $330 \mathrm{mg}$ of cocarbidopa. The mean duration taken for development of Augmentation in these patients was 2 months. There was no statistically significant correlation between development of Augmentation and mean blood sugar levels or with the duration of Diabetes in patients with underlying diabetic neuropathy. The symptoms of Augmentation reduced with treatment with Gabapentin in all 10 patients. The mean dose of Gabapentin used for Augmentation in all these patients to alleviate symptoms was $600 \mathrm{mg}$. Of the remaining 20 patients, there was a statistically significant reduction in IRLSSG score from baseline to that at 2 months $(\mathrm{p}=0.003)$ and at 6 months $(\mathrm{p}=0.009)$ while on co-carbidopa therapy. No significant side effects were documented due to co-carbidopa therapy.

\section{DISCUSSION}

Restless leg syndrome has a prevalence of $2 \%$ in Indian population 10,11 It is diagnosed with help of different clinical criteria. IRLSSG criteria is a standard accepted clinical criterion for restless legs syndrome and states that the following five essential points be met to be termed as restless leg syndrome-

1. An urge to move the legs usually but not always accompanied by or felt to be caused by uncomfortable and unpleasant sensations in the legs.

2. The urge to move the legs and any accompanying unpleasant sensations begin or worsen during periods of rest or inactivity such as lying down or sitting.

3. The urge to move the legs and any accompanying unpleasant sensations are partially or totally relieved by movement, such as walking or stretching, at least as long as the activity continues.

4. The urge to move the legs and any accompanying unpleasant sensations during rest or inactivity only occur or are worse in the evening or night than during the day. 
5. The occurrence of the above features are not solely accounted for as symptoms primary to another medical or a behavioural condition (e.g., myalgia, venous stasis, leg oedema, arthritis, leg cramps, positional discomfort, habitual foot tapping).

\section{Supporting Features}

RLS has a motor symptom periodic leg movements (PLMs) and also several clinical patterns that would support a diagnosis, especially when the diagnostic certainty is lacking.

\section{Periodic Leg Movements (PLM)}

PLMs are stereotyped, repetitive, withdrawal-like movements of the legs that occur when one is sleeping and so they are called periodic limb movements of sleep (PLMS). PLMs also happen during awake period. In contrast, PLMs during wakefulness, measured by suggested immobilisation test (SIT), has high sensitivity along with specificity for RLS, especially if patient has been evaluated many times and also in addition has subjective leg discomfort scores in multiple suggested immobilisation tests. To measure PLMs during wakefulness with SIT is another valid way to evaluate the severity and response of treatment in case of RLS.

\section{Treatment Response}

Many of the RLS patients do show at least minor initial clinical betterment from dopaminergic drugs or alpha 2 delta ligands, such as levodopa, dopamine agonists or Pregabalin. Large trials of diverse patient populations have showed good clinical response to dopamine agonist medication in around $60 \%$ of patients who participated. So, in general practice, an inability to respond favourably to dopaminergic drugs should raise doubts about accuracy of diagnosis but it does not always exclude a diagnosis of RLS. But, since non-responders to dopaminergic drugs exist but rare, compliance of the patient, and concomitant drugs that are taken by the patient should be checked.

\section{Lack of Profound Daytime Sleepiness}

Patients who suffer from moderate to severe RLS have long duration short sleep times but usually they do not report of daytime sleepiness which would be expected for so much of sleep loss. They usually have mildly elevated but normal Epworth Sleepiness Scale.

Local brain iron level is believed to play an important role in RLS pathophysiology. Decreased dopamine receptors are found in iron deficiency and it contributes to the deficient brain dopaminergic transmission ${ }^{12}$. Hence it was hypothesised that a relative dopaminergic dysfunction might lead to impairment in medial pain system and cause the uncomfortable symptoms noted in restless legs syndrome. ${ }^{13}$ In addition to this central theory, the increased occurrence of restless legs syndrome symptoms in patients with peripheral neuropathy led some people to postulate the role of peripheral nervous system dysfunction to contribute to restless legs syndrome symptoms. ${ }^{14}$

In a study by Allen et al 59\% of patients treated with cocarbidopa reported Restless leg syndrome associated augmentation effect. ${ }^{6} \mathrm{Hogl}$ et al reported that augmentation occurred in $60 \%$ of patients treated with co carbidopa. ${ }^{15}$ In our study augmentation occurred only in $33.3 \%$ of the patients treated with co-carbidopa. It was also noted that the mean co carbidopa dose in the patients who developed augmentation was $330 \mathrm{mg}(300+30)$ in study by Hogl et al. They also reported a median of 71 days for development of augmentation in a group of patients. These findings were in concordance to our study. These studies were performed in patients with idiopathic restless legs syndrome whereas in our study, a significant number of patients (24 out of 30 patients $-80 \%$ of study population) were having underlying Diabetic Neuropathy. Among the patients with chronic restless legs syndrome secondary to diabetic neuropathy only 6 of 24 patients (25\%) of patients developed augmentation. This goes on to underline the fact that incidence of augmentation might be low in patients with underling diabetic neuropathy. Among primary restless legs syndrome patients, 4 of 6 patients (66.7\%) developed augmentation. Our study could have been confounded by the low incidence of augmentation in secondary restless legs syndrome patients.

In our study we have used Max Planck Institute (MPI) Criteria for identification of augmentation. The core features of MPI criteria are-

\section{A. Basic features (All of which need to be met)}

1. The increase in symptom severity was experienced on five out of seven days during the previous week

2. The increase in symptom severity is not accounted for by other factors such as a change in medical status, lifestyle or the natural progression of the disorder;

3. It is assumed that there has been a prior positive response to treatment. In addition, either B or C or both have to be met:

\section{B. Persisting (although not immediate) paradoxical response to treatment}

RLS symptom severity increases sometime after a dose increase, and improves sometime after a dose decrease Or

\section{Earlier onset of symptoms ${ }^{8}$}

Recent treatment guidelines for treatment of restless legs syndrome do not favour use of co-carbidopa inpatients who fall under the category of chronic daily restless legs syndrome according to IRLSSG guidelines and instead advocate use of dopaminergic agonists. The reason stated for this being the high incidence of augmentation associated with co-carbidopa therapy. But since the incidence of augmentation in our study population is low and since the drug was effective in patients with chronic restless legs syndrome, it raises another important question whether cocarbidopa can be used as a first line agent in chronic daily restless legs syndrome especially in a resource limited country like India. It also emphasises on the need for local guidelines for restless legs syndrome therapy.

The treatment options available for augmentation include splitting up of dopamine agonists to a twice daily dosing or to change to an alternative dopamine agonist or to switch to an alpha 2 delta ligand (Pregabalin or gabapentin). In our study we have switched over the patients who developed augmentation to Gabapentin $300 \mathrm{mg}$ at bedtime. All the 5 patients who developed symptoms of Augmentation responded well to Gabapentin.

Of the 24 patients of secondary restless legs syndrome with underlying Diabetic neuropathy 10 patients were 
treated elsewhere for diabetic neuropathy with tricyclic antidepressants. The treatment offered in our centre consisted of stopping the tricyclic antidepressants (Due to the possible worsening secondary to anticholinergic properties) and starting on co-carbidopa. All patients reported prompt relief of their symptoms within first week of initiation of cocarbidopa as evidenced by increased quality of sleep and decreased night time symptoms.

\section{CONCLUSIONS}

Co-carbidopa may be an effective therapy in chronic daily restless legs syndrome as suggested by the good response in our study. The incidence of augmentation amongst South Indian population may not be as high as seen in western literature. Restless legs syndrome can be easily mistaken for small fibre neuropathy of Diabetes Mellitus and subsequent treatment with tricyclic antidepressants may worsen their symptoms. Hence correct identification of this treatable disorder becomes important to provide comfort to the suffering patient.

\section{REFERENCES}

[1] Garcia-Borreguero D, Silber MH, Winkelman JW, et al. Guidelines for the first-line treatment of restless legs syndrome/Willis-Ekbom disease, prevention and treatment of dopaminergic augmentation: a combined task force of the IRLSSG, EURLSSG and the RLS-foundation. Sleep Med 2016;21:1-11.

[2] Winkleman JW, Redline S, Baldwin CM, et al. Polysomnographic and health-related quality of life correlates of restless legs syndrome in the Sleep Heart Health Study. Sleep 2009;32(6):772-8.

[3] Earley CJ, Silber MH. Restless legs syndrome: understanding its consequences and the need for better treatment. Sleep Medicine 2010;11(9):807-15.

[4] Allen RP, Walters AS, Montplaisir J, et al. Restless legs syndrome prevalence and impact: REST general population study. Arch Intern Med 2005;165(11):1286-92.

[5] Ohayon MM, O'Hara R, Vitiello MV. Epidemiology of restless legs syndrome: a synthesis of the literature. Sleep Medicine Reviews 2012;16(4):283-95.
[6] Allen RP, Earley CJ. Augmentation of the restless legs syndrome with carbidopa/levodopa. Sleep 1996;19(3):205-13.

[7] Allen RP, Picchietti D, Hening WA, et al. Restless legs syndrome: diagnostic criteria, special considerations, and epidemiology: a report from the Restless Legs Syndrome Diagnosis and Epidemiology Workshop at the National Institutes of Health. Sleep Med 2003;4(2):101-19.

[8] García-Borreguero D, Allen RP, Kohnen R, et al. Diagnostic standards for dopaminergic augmentation of restless legs syndrome: report from a World Association of Sleep Medicine - International Restless Legs Syndrome Study Group Consensus Conference at the Max Planck Institute. Sleep Med 2007;8(5):520-30.

[9] England JD, Gronseth GS, Franklin G, et al. Distal symmetric polyneuropathy: a definition for clinical research - report of the American Academy of Neurology, the American Association of Electrodiagnostic Medicine and the American Academy of Physical Medicine and Rehabilitation Neurology 2005;64(2):199-207.

[10] Gupta R, Allan RP, Pundeer A, et al. Hindi translation and validation of Cambridge-Hopkins Diagnostic Questionnaire for RLS (CHRLSq). Ann Indian Acad Neurol 2015;18(3):303-8.

[11] Panda S, Taly AB, Sinha S, et al. Sleep-related disorders among a healthy population in South India. Neurol India 2012;60(1):68-74.

[12] Dauvilliers Y, Winkelmann J. Restless legs syndrome: update on pathogenesis. Current Opinion in Pulmonary Medicine 2013;19(6):594-600.

[13] Garcia-Borreguero D, Williams AM. An update on restless legs syndrome (Willis Ekbom disease): clinical features, pathogenesis and treatment. Curr Opin Neurol 2014;27(4):493-501.

[14] Gemignani F, Brindani F, Vitetta F, et al. Restless legs syndrome in diabetic neuropathy: a frequent manifestation of small fiber neuropathy. J Peripher Nerv Syst 2007;12(1):50-3

[15] Högl B, García-Borreguero D, Kohnen R, et al. Progressive development of augmentation during long-term treatment with levodopa in restless legs syndrome: results of a prospective multi-center study. J Neurol 2010;257(2):230-7. 